\title{
Narratives of the mystical among users of psychedelics
}

\author{
Abstract \\ We are now witnessing a radical revival in clinical research on the use of psychedelics (e.g. \\ LSD and psilocybin), where "mystical" experiences are at the centre. Drawing on in-depth \\ interviews with 50 psychedelic drug users, we document how they draw on archetypical \\ mystical narratives, comprising three key dimensions: (i) the transcendence of time and space; \\ (ii) deep euphoria; and (iii) the perception of being at one with "a larger whole." We suggest \\ that the evolving new cultures around the use of psychedelics contain a variety of narratives, \\ with clear roots in traditional mystical thinking. At the same time, these narratives reflect \\ current cultural and political influences, including the narratives of oneness with plants and \\ animals and our perceived need to protect nature. We conclude that the way people \\ experience mystical occasions due to psychedelic use have archetypical patterns, but \\ culturally specific storylines.
}

Key words: Psychedelics, LSD, psilocybin, ayahuasca, mysticism, narratives 


\section{Introduction}

Mystical experiences have been described throughout history. Classical sociologists such as Max Weber and Emile Durkheim devoted much work and thought to understanding the importance of such experiences (Tiryakian, 1995). Mystical experiences imbue the person with feelings of something sacred or holy, as well as feeling a deep level of unity (James, 1994 (1902)). The ordinary world is thought to vanish and replaced with something "pure" and "external". Recent empirical research on mystical experiences is to a large degree based on Stace's (1969/1987) sociological and psychological work on religious mystical experiences. A “complete” mystical experience implies e.g. "internal unity" (a merging with ultimate reality), "external unity" (all is one) and "noetic quality" (claim of intuitive knowledge of ultimate reality) (see e.g. Griffiths et al 2006, and Griffiths et al 2011).Whether these experiences emerge from religious ceremonies, intense meditation, or use of psychedelic substances, they appear to be an integral part of human history (Kelly and Kelly, 2007: 495), and naturally occurring psychedelic substances have played a significant role in the development of philosophy and religious thought in many cultures (Nichols, 2016). Even today, a large proportion of the general population (typically around one in three) report having had mystical experiences at some time in their lives (Hood et al., 2009).

It is no surprise then that people who use psychedelics such as lysergic acid diethylamide (LSD), psilocybin mushrooms, ayahuasca or N-dimethyltryptamine (DMT) often speak of their experiences using the language of the mystical or spiritual. Partly driven by promising new results in therapeutic use of psychedelics (Nichols, 2016; Pollan, 2018), there is now increasing interest in a better understanding of the mechanisms that bring about the therapeutic effects of these substances (Griffiths et al., 2006). However, while there are a number of clinical studies on the use of such substances, few sociologists have investigated 
how people experience and make sense of their use of psychedelics using interviews outside of clinical settings.

Using narrative identity theory as a framework, we examine how people living in Norway make sense of mystical experiences brought on by the use of psychedelics. Specifically, we document how they draw on stories rooted in political movements surrounding climate change and ecology to explain their experiences and their personal changes after using. We suggest that while there may be general and archetypical patterns to mystical experiences that emerge from psychedelic use, the cultural and situational environments of the users shape how they make sense of these experiences. The findings point to the importance of cultural narratives for making sense of mystical experiences and the personal changes that are associated with them.

\section{Psychedelics and Mystical Experiences}

Humans have long used psychedelics for mystical experiences. There is evidence for the use of natural psychedelics in the form of peyote cacti more than 5,000 years ago in Mexico (Bruhn et al., 2002). Eleusian ceremonies in ancient Greece were also most likely based on some form of psychedelic compounds (Wasson et al., 2008). In the Amazonian basin, ritual healing has been practiced for several thousand years, using ayahuasca, a drink combining plant-based DMT and monoamine oxidase inhibitors (Scultes and Hofmann, 1979). LSD became the archetypical psychedelic in Western society in the mid-20 $0^{\text {th }}$ century (Langlitz, 2013: 24-26). At the time, psychiatry lacked effective psychopharmacological medications, and several striking features of LSD use were emphasized (e.g. that users seemed to mimic symptoms of acute psychosis, such as ego dissolution and visual misperceptions). Moreover, users seemed to get into contact with repressed memories and the unconscious in a much 
swifter manner than with traditional psychoanalysis or psychodynamic therapy alone (Rucker et al., 2018).

LSD and to a lesser degree psilocybin were then used in psychiatric therapy and research from the mid-1950s until the mid-1960s, when the substances were put in Schedule 1 of the UN Convention on drugs. This change in scheduling seems to be due to two primary developments (Oram 2014). One reason was that new subcultural groups ("the hippies") began using psychedelics, creating a moral panic relating to the drugs (Goode, 2008). Another reason was the difficulties in conducting double-blind randomized studies to investigate the therapeutic efficacy of these substances. Norway experienced a similar historical development as the United States and European countries in the 1960s (Haave and Pedersen, 2020); Johnstad, 2020). As a result, there was almost no research on the therapeutic utility of these substances after 1965 until the 1990s when there was a revival of psychedelic research. This revival included new research projects initiated at universities in the United States and the United Kingdom that showed the value of these substances. For example, Griffiths and colleagues (2011: 662) found that a majority of healthy volunteers fulfilled the criteria for having had a "complete mystical experience" after being administered psilocybin, and four out of five of them rated this experience as one of the most spiritually significant experiences of their lives.

Numerous new research projects have now been initiated, and several of them suggest positive effects of psychedelics-assisted therapy on psychiatric conditions such as depression, obsessive compulsive disorder and nicotine dependence (for reviews, see: Johnson and Griffiths, 2017; Kyzar et al., 2017; Liechti, 2017). Recently, two new centres for studying psychedelics were opened at the prestigious universities Johns Hopkins in the United States and Imperial College in the United Kingdom. However, most who use psychedelics do so 
outside of therapeutic contexts. Even for this group, it has been suggested that the use may possibly have beneficial mental health consequences (Hendricks et al., 2015).

\section{Mystical Experiences and Narratives}

The key characteristic of psychedelics is that they may produce states of altered perception, thoughts, and feelings that are usually not experienced otherwise, often described as "mystical" (Nichols, 2016: 133). In this context, mystical is defined as "hidden meaning or spiritual power, causing feelings of awe and wonder" (Hornby, 1974). Freedman (1968: 335) reported that use of psychedelics was associated with "ego splits, the loss of boundaries, diminished control", and that "mystical or religious representations are apt for synthesizing these experiences". The reason is that "religion can relate man to his limits while taking account of his boundlessness" (p. 339).

Thus, the mystical seems to be important for understanding these experiences, and William James' (1994 (1902)) milestone study The Varieties of Religious Experience may be a starting point for identifying its primary dimensions. First, these experiences are ineffable. There is no positive way to describe them, in much the same manner as it would be almost impossible to describe the passions of love to someone who has not felt it. Still, the experiences are filled with obvious importance, they have noetic qualities, and may carry with them a strong sense of significance. Two further characteristics of mystical experiences are transiency and passivity: they usually last for a short time, perhaps only seconds or minutes, and they tend to engulf the person as though originating from an area outside ordinary consciousness (e.g. "a higher power"). A key question for James was related to whether these experiences typically are similar in their most important characteristics and across time and space and cultural contexts. 
Analytical philosopher W.T Stace (1987 (1969)) sought to establish such a generic or universal core, sampling mystical experiences from Christian, Islamic and Buddhist religions. He observed that those with these experiences used a highly figurative language that "added something to the experience". This language helped to understand it by giving it "classificatory concepts, logic inferences or explanatory hypotheses" (p. 37). He suggested key characteristics, overlapping those of James, of these experiences: First, a sense of "objectivity", resembling the "noetic" dimension. Second, a strong "positive affect". Third a feeling — closely related to the former — of something "sacred or holy". Moreover, Stace argued, the deepest mystical experiences implies the experience of unity. The ordinary perceptual world is abolished, opening up for a "pure and unitary consciousness" (p, 87). Metaphors such as "melting", "loss" and "death" are common when explaining these experiences, and for example Sufis (Islamic mystics) have a special term for this, fana, meaning "passing away" (Stace, 1987 (1969): 93). Both James and Stace suggested that even if there are diverse expressions of mystic experiences, they still have some uniform characteristics.

The radical constructivist philosopher Steven Katz (1978: 26) is the key voice arguing that there are no "universal", "pure" or "unmediated" mystical experiences. He argued that all such experiences are processed, made available to, and categorized by means of epistemological processes with roots in a concrete upbringing, culture and religion. Thus, an Islamist is not able to escape her upbringing in Islam, and a Jew will have Jewish-type mystical experiences. However, this position has met criticism, as mystical experiences often have occurred in naïve persons with no previous engagement in any forms of religious life (Kelly and Kelly, 2007: 516). The experiences also seem to have striking similarities to other mental phenomena. For example, uniform sleep-paralysis experiences observed in different 
parts of the world seem to reflect a likely corresponding uniformity in the psychological, physiological or biological conditions under which these experiences occur (Hufford, 2005).

In recent empirical research on mystical experiences occasioned by use of psychedelics from psychiatric and psychopharmacological milieus (e.g. Griffiths et al 2006, MacLean et al 2011), there are no references to Katz' constructivist perspective. When identifying the elements of the "complete mystical experience", some researchers briefly refer to social scientific research from the 1960s (e.g. Stace, 1969/1987) but they do not try to link such experiences to cultural storylines from participants' daily lives. Interestingly, several contemporary researchers are aware of the powerful effects of set and setting in clinical use of psychedelics. Thus, they prepare sessions carefully and offer interpersonal support during the period of drug action (Griffiths et al 2006: 269). However, they are not preoccupied with the broader narratives underlying their participants' experiences during the trips.

Assuming that mystical experiences are indeed mediated, we seek to understand how people make sense of mystical experiences that are not affiliated with specific religions. Specifically, we seek to determine how young adults in Norway make sense of mystical experiences brought on through the use of psychedelics by examining their stories relating to these events and to their personal change within a specific cultural context, which emphasizes ecology and downplays religion.

\section{Narrative sociology}

The idea of mediated mystical experiences is consistent with ideas from narrative sociology. A fundamental characteristic of humans is that we use stories to make sense of the world and of ourselves (Holstein and Gubrium, 2000). Indeed, even our physical senses such as seeing, hearing, and feeling are often incomplete and we fill in the missing information through internal storytelling (Storr, 2019). This reliance on stories is likely true for psychedelic 
experiences as well. The ineffable and short lived nature of psychedelic experiences lend themselves to storytelling. The feelings and emotions brought forth by psychedelic experiences are difficult to process, understand and express, and through storytelling we can make these experiences "real" or at least relatable to others. By connecting the experiences to more commonly known ones we can make sense of and explain our experiences, including those relating to personal change. The use of analogies and metaphors help us make the ineffable tangible. As such, a narrative identity framework may be well suited to understand the stories of those who experience the mystical that results in personal change when using classic psychedelics.

Narrative sociology suggests that stories are essential elements of culture that people use to make sense of their lives. People use stories to shape, inspire, and uphold behaviour. While we try to make stories unique to ourselves, it is assumed that people act out cultural stories when they engage in behaviours or related experiences (Presser, 2009). Narrative thought encompasses a variety of perspectives to study narratives. Here, we interpret our findings using a version of narrative theory that focuses on the role of narratives in creating personal and social identities (Loseke, 2007). According to Loseke (2007), narrative identities occur at three levels: macro-level (cultural identities), meso-level (institutional and organizational identities), and micro-level (personal identities). Narratives at the macro- and meso-level shape cultural identities, which include "the imagined characteristics of disembodied types of people that simplify a complex world and construct symbolic boundaries around types of social actors" (Loseke, 2007: 663). The groups created with cultural narratives are broad social classifications of abstract actors who represent generic social types. When referring to people who use illicit drugs, cultural narratives reflect the larger cultural assumptions about these people, which typically condemn those who use ( Thus, we would expect that the specific narratives of those who use psychedelics (including 
how they experience the drugs and their mystical experiences) are shaped to some degree by such larger cultural narratives.

Identity narratives at the micro-level are those that help shape personal identities. They are the specific ways that people construct personal self-understandings and present themselves publicly (Loseke, 2007). Personal narratives are created by taking abstract, depersonalized narratives (i.e. cultural and organizational narratives) and adding complexity, individual histories and personal perspectives to the narrative to make them specific to the person telling the story (Sandberg, 2013). The details and specificity added to cultural or organizational narratives allow people to create a coherent self that is unique yet connected to a more abstract group. These larger narratives aid in explaining the ineffable.

Using this framework, the aim of the present study was to investigate how people draw on cultural narratives to describe the mystical experiences related to use of psychedelics and to make sense of personal changes in their lives. Doing so can shed light on the mediated nature of mystical experiences.

\section{Methods, data and analysis}

To understand the mediated nature of mystical experiences and psychedelics, we draw on semi-structured interviews with 50 Norwegian men and women. To fit the sampling criteria, participants had to have some experience with psychedelic substances. Participants were mostly recruited through a Norwegian online forum for users of psychedelics (called Emmasofia), some were also recruited by means of snowball-sampling based on these initial participants. Participants' area of residency differed but the majority were living in the greater Oslo area.

Interviews were carried out by the first and third author in our offices at the University of Oslo. We tried to develop a friendly and relaxed atmosphere and interviews lasted between 
two and three hours (usually with a 10 minutes break). We asked about topics such as the participants' upbringing, family background, education and occupational career. We also explored their political interests, food preferences (e.g. vegetarianism or veganism) and taste regarding music and literature. In addition, we asked about details of their history of use of alcohol and illegal psychoactive substances. Then, the greater part of the interviews addressed their use of psychedelics. In this section, we took particular care to pose open questions, which allowed participants to provide rich stories with numerous details about inspiration, motives and the social and sociocultural contexts of using psychedelics. They described substances, rituals, typical dosages, and offered in-depth descriptions of their trips. Some had taken part in well-organized ceremonies, including those with shamans flown in from South America, but most had used them in more informal contexts at festivals, private homes or social gatherings in the forests surrounding Oslo.

All demographic information were self-identified by the participants. We interviewed 42 men and 8 women. The age of participants ranged from 20-55 years, but the majority were in their late twenties or early thirties. A few had used psychedelics less than ten times, but the majority had used them between 10 and 50 times. The participants had experience using LSD $(n=37)$, psilocybin $(n=36), 2 C-B(n=20)$, DMT or ayahuasca $(n=20)$, and MDMA $(n=$ 34). Participants varied as to whether they considered MDMA "a real" psychedelic. ${ }^{\text {i Some of }}$ these users had also experiences with "microdosing" (regularly taking small doses of LSD or mushrooms) (Johnstad, 2018, Webb et al 2018). However, this was not a prevalent pattern in this sample. Almost all use of psychedelics was illegal, the exception was a few experiences taking place in Netherlands, where the use of psilocybin truffles is semi-legal (they may be bought in smartshops in Amsterdam). However, few of these users were concerned with the illegal dimension of the use. This may be due to the fact that the Norwegian police force has not prioritized policing the use of these substances. Moreover, due to the low prices and the 
low-frequent use as compared with other illegal substances, the illegal economy surrounding psychedelics is not much interwoven with traditional drug crimes.

All participants were students or worked full-time, and their educational level was above Norwegian average: $70 \%(\mathrm{~N}=35)$ had or were in the process of completing academic degrees ranging between bachelor's to $\mathrm{PhD}$. Many described their experiences drawing on sophisticated philosophical, cultural or literary references, reflecting high levels of cultural capital. Many also were knowledgeable on hallucinogenic substances and they often referred to scientific studies to document their stances on the matter. Users of psychedelic substances seemingly have little in common with those who use cocaine, heroin or amphetamines in regards to sociocultural style, use of symbolic boundaries, and identity work. Almost all of the participants had also used cannabis during the preceding year, whereas a large minority (between 30 and 40\%) had used other illegal substances, such as amphetamines or cocaine, figures that are much higher than those observed in the general population in Norway (Norwegian Institute of Public Health, 2018). Still, they lived well-organized lives, and none of them they had substance use problems. We are not able to estimate the prevalence of psychiatric disorders in the sample; however, a majority of participants said that they had struggled with problems such as depressive periods, sleeping problems, generalized anxiety, or misuse of alcohol or illegal drugs. Some had received therapy for these problems.

The interviews were audio recorded, transcribed, and then coded using NVivo, following general standards of qualitative research analysis (Kvale and Brinkmann, 2009; Silverman, 2009). Specifically, we used thematic narrative analysis to understand participants' mystical experiences using psychedelics. Our initial coding for mystical experiences included coding long sections of text so that the broader narratives could be identified. We analysed their narratives in a way to ensure that we looked at their stories in their entirety (i.e., stories were the unit of analysis). During this stage we determined the 
contours of their experiences, and how these experiences were connected to broader cultural narratives and to their personal narratives. We took care to draw from a large portion of the participants to better illustrate the generality of these experiences.

It is true that characteristics of the interviewers and participants (e.g. age and class) as well as the interview setting can have an effect on rapport and shape how people respond to questions. For example, many participants in this study referenced social scientific and philosophical literature in their narratives, which may have been encouraged by the interview context at the university and the fact that the first author is a publicly well-known researcher in Norway. We are unable to determine whether and to what degree the stories of participants were influenced by such factors. However, the similarity in participants' stories with research on mystical experiences broadly and relating to psychedelic use suggest positionality and setting had limited effect on participants' stories. Additionally, we are interested in the way participants narratively make sense of their experiences using psychedelics, rather than on factual recounting of their experiences.

The study was approved by the ethical review board of Norwegian Social Science Data Service (NSD) on behalf of the Norwegian Data Protection Authority (project 445634). Participants gave their active informed consent. Identifying information (including geographical references, names of partners and friends), were removed and replaced with aliases.

\section{Cultural Narratives and Experiencing psychedelics}

The participants described their experiences using psychedelics in ways that mirrored findings from other research. Namely, they spoke of the difficulty of describing their experiences but found ways to discuss the transcendence of time and space, feelings of euphoria, and being connected with a larger world. In what follows we elaborate on these experiences and how they drew from larger narratives in attempts to explain the ineffable. 


\section{Talking about tellability}

Psychedelics can lead to experiences that are unlike those brought on by other drugs. Participants described that their personal relationships changed, including how they felt deep empathy and perceived closeness with others. Many described "bad trips" that in the moment were terrifying, but with time interpreted as leading to deep existential insights. They described psychedelics as bringing about ego-dissolution and a subsequent sense of connectedness with the world. But, explaining these feelings was difficult for them. Maria, who was an experienced user of psychedelics, said:

The sentence you'll most often will encounter in this project is that, "It's very difficult to explain, but ..." Followed by a few sentences where they'll try to explain it anyway. But it is extremely difficult to explain. ... You know what you're saying is pretty meaningless. But you can try, of course, try to get to grips with the narrative around it.

Despite being difficult, Maria and other participants explained the experiences through storytelling, analogies, or metaphors. The importance of metaphors was directly mentioned by Felix:

The inner/outer dissolves. I find myself melting into a stone. I'm left thinking: inner/outer. They're metaphors, of course. All language consists of metaphors. I find Richard Rorty and George Lakoff very persuasive in that the way we think consists of bodily, situated metaphors. Inner/outer, this isn't a truth but a useful piece of fiction. 
When telling their stories our participants borrowed from science, philosophy and fiction, and more general experiences to help others "get" what it is like to use psychedelics. Many of these stories contained statements about how the users changed due to their experiences with psychedelics. The analogies they called forth differed but the larger idea was to connect to the listener by using shared, recognizable experiences. Several explained their mystical experiences by relating them to religious transformations. While also ineffable, these types of stories are known among most people (Ammerman and Williams, 2012). As Harry said:

The feeling was the same as described in religious scripts and the experiences some of them portray. Classic psychedelics can allow you to access this. It's difficult to put the feeling into words, but "awe" comes close.

It is difficult for people to describe mystical or transformative experiences. When attempting to describe these feeling, participants used relatable stories to facilitate the explanation. While participants varied in how they sought to relay their experiences with psychedelics, they did plot their stories with similar themes. In what follows we discuss participants' mystical experiences that arose from using psychedelics. We begin with their stories of the more concrete experiences; namely, how they transcended time and space and the enhancement of mood and euphoric feelings that come from use (even once the hallucinogenic effects go away). We then proceed to the deeper, mystical level of the experiences, and discuss how they described this as being at one with a larger whole, which centred on nature and ecologys.

\section{Transcendence of time and space}

The most common description of psychedelic trips among participants was that it led to them feeling a transcendence of time and space or a "concentration of time" (Watts, 1968). This 
meant a slowing down of the perception of time where short moments felt as if they lasted for hours. Amelia described such an occasion after using psilocybin mushrooms with friends and they were going to a pub afterwards:

We took some magic mushrooms and then went to the pub. After we'd paid, the waitress was supposed to come back with our change. We started looking at each other, wondering “How long have we been sitting here?” Perception, time, distances, quantities may all be blurred out.

Edward spoke of the general feeling of time while using. As he said, "We humans are in equilibrium because you can always check yourself against the time, whatever that may be. 'Now an hour has passed by'. Imagine a world where you have no sense of time. A moment feels like an eternity."

Others had deeper connections with the transcendence of time and space than simply not knowing how much time had passed since using. These participants spoke of how they transcended time and place implying that their individual selves opened up for a more altruistic understanding of themselves and their loved ones. John spoke of the simultaneously collapsing of time and space and individual identity and how it connected him with his family.

I felt that I was them [my father, mother and brother]. I could see them starting off as children and growing older, older and older, quickly, in a few seconds. I felt all the feelings they felt in that period of time. 
Thus, merging with his closest family members, John afterwards felt a unity with them that he had never felt before. Typically, perceptual distortions involving time and space may be experienced as threatening (Lloyd et al., 2012). However, among our participants, they often opened up for empathy and feelings of unity. Echoing this, Noah felt connections with all of humanity.

I found myself on the savannah one hundred thousand years ago and was part of the first human evolution. We were ape-like beings, dancing round the bonfire. I was there and I was reborn and died and born anew. The roots of all mankind were there.

Noah was taken back to the start of humanity, in a voyage through time and space, but also to the geographical roots of mankind, thought to be located at "the savannah". The transcendence of time was frequently mentioned with the collapsing of space. They spoke of their sense of being able to transcend space and be at many places at once. According to Samuel:

You vanish completely into the other world. The man who was with me [during the trip] said he was on a savannah in Africa. He could see the savannah stretching out far before him and he was slowly vanishing into that savannah.

Note how Samuel used the same concept as Noah; for both "the savannah" is taken to geographically represent the roots of mankind. However, Samuel was even more concrete than Noah, and located it in Africa, reflecting a standard current historical narrative of the origins of humans (Stringer, 2003). Their connections were with the past, but also with the 
land and humanity. Rather than connecting with an otherworldly plane, they were grounded to Earth.

\section{Positive mood/euphoria}

Previous studies suggest that psychedelics may have paradoxical effects: One the one hand, they may elicit unpleasant psychosis-like reactions, yet they are often linked to strong psychosocial wellbeing (Carhart-Harris et al., 2016). Our participants described this ambiguity —on the one hand, unpleasant bad trips, at the same time profound euphoria (Gashi et al., 2020). These euphoric feelings transcended their daily feelings of happiness. While euphoria can be experienced with other drugs as well, participants said psychedelics came with a much deeper and existential sense of happiness. Maria used MDMA as a contrast when articulating the euphoria she experienced when using "real" psychedelics.

It's wasn't like the euphoria you get from MDMA. The euphoria from MDMA is nicely euphoric. Like lying in a Jacuzzi with a glass of wine and getting a massage. The euphoria from psychedelics is more like standing on the top of a mountain, looking at the ocean, woooo! I haven't had any children yet but I bet it's like the type of looking-at-your-first-kid-euphoria.

Maria echoed several participants in that MDMA was regarded as a more "simple" substance than the "real" psychedelics. Indeed, MDMA could open up for intense feelings of empathy, and is therefore sometimes characterized as an "emphatogen" (Hysek et al., 2014). The descriptions of use of MDMA echo the effervescence experiences described in religious ceremonies by Emile Durkheim (Durkheim, 1995 (1912): 216-25). However, MDMA had more superficial effects than the "real" psychedelics, several argued. 
George explained the euphoria he experienced after taking LSD by saying it was like, "Being in it completely, no questions, no fears, no doubts. The feeling of returning to something familiar. The feeling that things are more interconnected than you'd believed, and a very powerful feeling of love. Everything was so incredibly tactile, genuine, living, breathing, vibrant."

The bliss experienced by participants seemed to be linked to the temporarily freedom from the exhausting self-reflexivity of the ego. It has been suggested that it is precisely the time-out like freedom linked to ego-dissolution and this self-less type of consciousness that is also the key to the therapeutic effects of psychedelics (Pollan, 2018: 271).

The feeling of euphoria and contentment was strong for many participants. In fact, some, like Peter, said that the feelings they got from using made their lives complete. As he said:

Then I found myself thinking that perhaps I would die in a few months, but also that "that was fine. It was worth it". Because this is the greatest thing you'll ever experience. You've experienced everything. "My life is complete, now I can die". You can see the cornerstones of the religions: how everything is interconnected.

These descriptions echo Watts's (1968) early formulations of how the users of psychedelics felt being content with death and with life. Participants told that psychedelics brought about deep existential experiences, similar to what is previously classified as mystical. These experiences were the ones that brought about "true" change in their lives, they argued.

Being at one with "a larger whole" 
Our participants often saw themselves as part of something larger, feeling connected to "the universe". Often their stories built up to an existential climax where where descriptions such as feeling that "all is one" were typical. As Charles said:

The way I see myself is that I just am here on this planet now. I can see myself as part of something bigger. ... Everything is drawing together, converging. I can see, especially in relation to my parents and what the future holds, into the future.

The interplay between seeing oneself as part of something bigger, such as "the universe" and emphasizing significant others, such as partners and family members, was typical. In a similar vein, John described what he could experience at the height of a trip:

Partly abstract experiences and partly specific images. Then the images started to transform into members of my family. I saw my father, I saw my mother, I saw my brother. I could see them but I also experienced being them.

Thus, sometimes an ego-death or dissolution was linked to the person merging with close relatives, typically significant others, for John it was his parents and his brother. He not only saw them, he also became them, and he experienced and perceived the world and the surroundings from their perspective.

None of our participants described themselves as active Christians, even if some had a Christian background, sometimes leading to an openness for the divine. Still, several told us about "God-like" supernatural instances. Jonathan said: 
Suddenly there's a clear voice resounding from above. It must have been "God", right? But it wasn't God; definitely not. I think it's because I'm an atheist [chuckles]. There was a dialogue and although both voices were me, I controlled only one of them. Only the first one. The other one was new and it was pretty accusatory, bossing me about in a way. Since then I've realized that I often talk to myself in that way [chuckles]. I $d o$ often have that voice in my head.

The key feature in his narrative was that the seemingly supernatural instance, "God", was discursively undermined by one part of Jonathan himself. He perceived himself as an atheist, and he linked this characteristic to a dialogue where he was in command of one voice, but where the other-which was not "God"-still criticized him and "was bossing him about". Gradually he realized that he often talked to himself in this manner.

The underlying theme in these experiences - to see oneself as part of "the universe"may be situated in the heart of the classic literature on psychedelic mysticism: Participants describe how their ego is lost (Leary et al., 1964) or dies (Grof, 1980), and they become part of something bigger. However, when concretizing this experience, they often used references from their everyday surroundings, and even characteristics of themselves, such as in Jonathan's story.

Moreover, they also drew on tropes relating to the climate crisis, ecology, nature and plants, which are keys issue in current public debates in Norway (Bohm et al., 2016). They used these themes to ground their experiences and make them understandable. Often this was expressed in the closeness to nature. As George said:

I got this powerfully heightened sensitivity to nature. I felt like nature had a consciousness unlike humans, but it's some kind of living part of the earth. Reaching it 
produced a feeling of such tremendous awe, just like "shit! Mud and roots. Nature is awakening after winter". It was just like "wow!"

Suzanne, in a similar vein, emphasized how the concrete experiences of trees and berries opened up for a kind of animistic perspective, typical in tribal religions, where objects, places and creatures all possess distinct spiritual essence, but which is also reflected in the so-called "animistic turn" in archaeology and anthropology (Haber, 2009). Suzanne said:

Many things in the forest start talking to you. I like climbing trees. [Chuckles] You become a different person, a playful one. You become at one with nature. You can just lie in the heather eating blueberries and have blueberries all over your face. That's in a way part of the fantastic thing about it. You get to meet people who just are.

Participants explained to us how magic mushrooms, mescaline and iboga had been used for spiritual purposes for many thousand years. They were often interested in indigenous knowledge, and emphasized the respect owed to local beliefs and practices as well as to shamans coming from Latin America. A key reference for many was Terence McKenna's (1992) Food of the Gods, where psychedelics are understood in a historical perspective and from a shamanic understanding, linked to reverence for nature. Anna told us:

The plants show you how to do it. Thanks to them, you reach parts of your subconscious that are deep, deep down. If you're up here and your dreams are down there, you get the feeling that there's something outside of your self talking to you, because it's so deep. And the plants showed me that I'd been living my life wrong. How could I live it differently? By growing plants. 
Peter as well told how his use of psychedelics was linked to growing his own plants as well as having to learn to live in nature. He got inspiration from his Sami ancestors:

I started growing plants. I went out walking in the great outdoors, it was December, and I left the paths. I could communicate with nature in a completely new way. I had to learn to live in nature. But how was I to do that? I didn't know anyone who knew how to do that, apart from [people] in the Sami culture. Maybe I should try walking on all fours? How was I to be in nature now?

Felix was well read in philosophy and described, in a rather elaborate argument, how his mystical experience was inspired by Nietzsche:

We're competing with each other because [the world's] resources are finite. But we shouldn't hate each other. I was tripping in the forest, thinking: what if an animal appears, a predator that pounces on me right now? I'd kill that animal if I had to but I'd do it out of a kind of love. Not out of hate, but out of necessity. I'd call that a Nietzschean mystical experience. It's not that idealistic "we all have the same goal and can just join hands and be friends". But we can compete with one another with more goodwill. We shouldn't hate each other even though we're competing.

Felix referred to Nietzsche, a philosopher who has gained increasing popularity among scholars studying mysticism. Several refer to his term Unwissenhet. The concept point to the calmness that may arise when one escapes the pressure of neverending self-reflexivity (Smith, 2016). 
However, the most important and common denominator in these narratives was how a Zeitgeist centring on nature and ecology was reflected in our participants' mystical experiences with few similarities to the neoliberalist discourses described in a study among users of psychedelics less than two decades ago (Riley et al., 2010). This development may perhaps be understood through the sociological term "political ecology", borrowed from environmental sciences and suggesting a link between ecology and political engagement that is currently observed in large cohorts of young people in many parts of the world (France and Threadgold, 2016). It also allowed them to make sense of these mystical experiences in a way not directly tied to traditional religion, which was important for the largely non-religious sample. Rather than drawing on religion (i.e., Christianity), our participants drew from more secular cultural stories prominent among their social positions in Norway when making sense of their own experiences with psychedelics. Their emphasis on closeness with nature is part of a traditional Norwegian preoccupation with nature and outdoor activities (Gurholt and Broch, 2019). However, the stories of our participants also reflected an increasing political and cultural emphasis on ecology, a threatened nature and a concern for environmental issues. They were preoccupied with how biodiversity loss threatens human well-being and possibly human existence (Skogen et al., 2018). Many of our participants sympathized with activists such as climate activist Greta Thunberg, who was awarded name of the year by Time in 2019 . However, one should note that previous studies of users of psychedelics, from other contexts, have also observed increased attachment to nature and the environment when compared with those who use cannabis, amphetamines or heroin (Lerner and Lyvers, 2006). In a small-sample mixed-model design study, Lyons and Carhart-Harris (2018) found that the use of psilocybin was related to lasting increases in feelings of being connected to nature. Similarly, Forstmann and Sagioglou (2017) found that previous experiences with classic psychedelics predicted engagement in pro-environmental behaviours, and that that the 
relationship was statistically "explained" by people's degree of self-identification with nature. Thus, our findings reflect previous studies that suggest users of psychedelics in a variety of contexts may also make sense of their mystical experiences by emphasizing connectedness with nature.

\section{Personal Narratives and the Psychedelic Experience}

Personal identity narratives (those that tell to define who we are as people) are best understood in the relation to cultural narratives (Loseke, 2007). Thus, when explaining the ineffable experiences of using psychedelics the participants drew on larger cultural narratives common in Western cultures. Specifically, they drew from narratives relating to ideas about the importance of ecology and connections with the environment rather than religion when making sense of their experiences. But these narratives do more than give metaphors for understanding their experiences. They also shape people's personal identity narratives (Loseke, 2007). Participants used their descriptions of their experiences to also present themselves as certain types of people - those who were bold enough to try new experiences and who came away as better people for it. Thus, these narratives helped shape how participants defined themselves and presented themselves to others (at least during the interviews).

One of the primary ways participants identified themselves in relation to their use of psychedelics was as people who were in tune with themselves and with nature. They spoke of how these mystical experiences led to a greater sense of belonging than they once had. For many these feelings became a way they described their transformations. They believed that their mystical experiences associated with psychedelics led them to be better people. Peter said of his new identity: 
These plants [mushrooms] show you how you should live your life. Because you get in touch with parts of your sub consciousness that are otherwise not visible. So, you'll get the feeling that it is something outside of yourself that speaks to you - it is that deep. For me, it showed me that I have been living and doing it the wrong way, it has taught me how to live differently. My whole life have been about technology. Then [after psychedelics] I saw what it was, and what it did to me, I saw the imbalance in my life and I learned how to balance it.

Similarly, Axel spoke of his change to a better version of himself. He described meeting a god-like creature who confronted him, which led to personal change for the better. Seeing such life-changing entities has been associated with psychedelics experiences (Davis et al., 2020). As Axel described:

To experience or meet god, has made me much more humble to my own knowledge, of what I know and what I don't know. I've never believed that I have all of the answers to things, but now I realize so much more how far I am from having all of the answers. I've improved, and I've done changes in how I think, perceive stuff etc., which have made things better for me and the people I interact with.

Others participants said that they found an inner peace that allowed them to see other people in more positive ways as well. They said they became more accepting and trusting of others. Instead of looking at fault in others, they accepted them as they are. For Martin, this meant becoming more open with people. As he said:

Psychedelics make people more open, and I've definitely noticed that within myself: I judge people a lot less now. I grew up with a brother that is very judging, and I've kind of mirrored that, he is my big brother, right. He has a very narrow category of who are 'acceptable" and okey, and if you're not part of that narrow category, then you get judged by him. I'm done with that, and now I'm like: as long as you don't do 
damage to people or the world, then it should be okey for people to dress and appear how the heck they want. So, I'm much more open like that, and I have stepped out of his [the brother] shadow now.

The personal narratives of the participants emphasized their growth as people. As such, they differed from the typical tragic drug stories of meetings with dark forces in modern Western society (Sandberg and Tutenges, 2015). Whether they saw others in more positive light, they gave up toxic parts of their personality, or they found a better balance in their lives, many spoke of positive changes brought on by their psychedelic experiences. These changes were connected to their larger descriptions of their mystical experiences. Namely, they spoke of personal change that was consistent with a belief in the importance of being a part of a larger whole. Such stories allowed them to align past failures or mistakes with current versions of themselves. Using personalized narratives of their experiences they were able to provide a coherent connection among their life events. They were more than the stereotypical "hippie" psychedelic users or those who use purely as a party drug. They were people who grew and were self-aware. They were people who were aware of the interconnectedness of the world and how they could contribute to this.

\section{Discussion and Conclusion}

Participants in our study perceived it as difficult to describe the experiences brought on by psychedelics, echoing the ineffability dimension typically described by previous scholars (Stace, 1987 (1969)), but were clear that these experiences changed their lives for the better. Key aspects of their psychedelic experiences were transcendence of time and space, ego dissolution or death, and pronounced euphoria, phenomena that are described in the clinical literature as well (Griffiths et al., 2006; Langlitz, 2013). However, they also made great efforts in trying to explain to us what must be considered a "mystical core" of what they had 
experienced. Their narratives when trying to do this, were ambiguous, and suggested on the one hand universal or generic dimensions of the mystical. At the same time, they were influenced by contemporary cultural streams and political preferences, or what may also be classified as typical macro level, cultural narratives (Loseke, 2007). The general pattern of their experiences (from the ineffable quality, to the transcendence of time and space, to the mystical level) is consistent with decades of research on psychedelics. However, the specific nature of the stories they told, from analogies and metaphors to the details of their perceptions, can be interpreted in the light of these participants' cultural and political environments. Generally, they were preoccupied with ecology, climate crisis, plants and nature. They were worried about the physical transformation of nature. Such cultural beliefs certainly shaped their perceptions and descriptions of their mystical experiences. These larger narratives also shaped how they presented their personal narratives. They believed that these mystical experiences made them better people. They experienced a change in perceptions of their selves (and of others) that made them more connected with the larger world.

A key controversy in the debates around mysticism is related to the question about whether the experiences have uniform characteristics, if there are "pure" or "unmediated" experiences (Katz, 1978; Stace, 1987 (1969)). The feminist philosopher and theologian Graze Jantzen (1995) critically dissected the ways in which modern constructions of mysticism had "privatized and domesticated" it, in particular the lack of investigating the possible connections to power and gender. She criticized the ways politics, materiality, embodiment and social ethics was neglected, claiming that mysticism is never essentially this or that, but rather "a constantly shifting social construction" (p 24). Philosopher Steven Wasserstrom (1999), in a similar vein, criticized the abstract essentialism of the postulated mystic, coining the academic fixation here as "mystocentrism". Thus, they positioned themselves in the tradition of Steven Katz (1978), arguing that there are no unmediated mystical experiences. 
In a previous study linking experiences with psychedelics to a contemporary cultural frame, Riley et al (2010), drawing on a sample of users of psychedelics from the United Kingdom and data from 2004, identified an overarching discourse of neoliberalism, described through keywords such as "the right to exercise personal freedom" and "economic citizens". Here, use of magic mushrooms was an occasional leisure activity in a context of plentiful economic resources among people often participating in urban nightlife. The experiences of the contemporary Norwegian users of psychedelics differ from these findings. However, they echo a handful of studies, primarily from the United States, that show a link between the use of psychedelics and increased concern for nature and ecology (Lerner and Lyvers, 2006; Lyons and Carhart-Harris, 2018). One should also note that during the first wave of use of psychedelics, the hippies were deeply engaged with nature and ecology (Wesson, 2011).

While previous research has shown connections between psychedelic research and connectedness with the environment, this is not the case for all who use these types of drugs. Psychedelics are also used among those who attend raves and other party settings (FernándezCalderón et al., 2018). Those who use psychedelics solely in these party settings seldom speak of the mystical properties of use. They discuss similar effects (e.g., connectedness and ego dissolution) but not in the context of the mystical. Rather, they emphasize the bonding and connections among users (Perrone, 2016). This suggests that the context of use may be important for how people interpret or make sense of their psychedelic experiences.

In addition, our participants' experiences were surprisingly similar to those described in the classical literature on mysticism, pointing to the ineffability in describing them and the transcendence of time and place. However, we also noticed an ambiguity. On the one hand, participants described the classical identifiers of the mystical experience. They described experiencing "awe" when meeting the roots of the universe and unity with a larger whole. At the same time, the way they described their experiences were highly coloured by their 
immediate surroundings (e.g. by their relations to family and partners). An even more striking feature was the strong link to the most important theme in the current political debate in Norway, with an increasing emphasis on climate, ecology, nature and plants. The participants did not draw on religious themes to make sense of their experiences. Rather, the largely nonreligious group drew from secular themes relating to the environment and ecology and their place in this system to tell their stories.

Such an understanding of the experiences of psychedelic use is consistent with current understandings of narrative identity theory. That is, while people are storytellers, we do not create stories from scratch. Instead, we draw on pre-existing themes and tropes to shape our stories. We take from the abstract, dis-embodied stories and integrate personal details to make them specific to our own experiences (Loseke 2007). Narratives and stories aid in explaining the ineffable, but they also do more. Stories allow us to make sense of our own experiences. As such, our findings show support for the mediated nature of mystical experiences and illustrates how narratives both explain and shape these experiences. 


\section{Reference List}

Ammerman NT and Williams RR. (2012) Speaking of methods: Eliciting religious narratives through interviews, photos, and oral diaries. Annual Review of the Sociology of Religion 3: 117-134.

Bohm G, Pidgeon N, Poortiga W, et al. (2016) European perceptions of Climate Change, Oxford: Climate Outreach.

Bruhn JG, De Smet PA, El-Seedi HR, et al. (2002) Mescaline use for 5700 years. Lancet 359: 1866.

Carhart-Harris RL, Kaelen M, Bolstridge M, et al. (2016) The paradoxical psychological effects of lysergic acid diethylamide (LSD). Psychological Medicine 46: 1379-1390.

Copes H, Hochstetler A, Williams JP. (2008) “We weren’t like no regular dope fiends": Negotiating hustler and crackhead identitites. Social Problems 55: 254-270.

Davis A, Clifton JM, Weaver E, et al. (2020) Survey of entity encounter experiences occasioned by inhaled N,N-dimethyltryptamine: Phenomenology, Interpretation, and Enduring Effects. Journal of Psychopharmacology. Online publication doi.org/10.1177/0269881120916143

Durkheim E. (1995 (1912)) The elementary forms of religious life, New York: The Free Press. Fernández-Calderón F, Cleland CM and Palamar JJ. (2018) Polysubstance use profiles among electronic dance music party attendees in New York City and their relation to use of new psychoactive substances. Addictive behaviors 78: 85-93.

Forstmann M and Sagioglou C. (2017) Lifetime experience with (classic) psychedelics predicts pro-environmental behavior through an increase in nature relatedness. Journal of Psychopharmacology 31: 975-988.

France A and Threadgold S. (2016) Youth and political economy: Towards a Bourdieusian approach. Jornal of Youth Studies 19: 612-628. 
Freedman DX. (1968) On the use and abuse of LSD. Archives of General Psychiatry 18: 330347.

Gashi L, Sandberg S, Pedersen W. (2020). Making "bad trips" good. How users of psychedelics narratively transform challenging trips into valuable experiences. International Journal of Drug Policy. Early online publication doi.org/10.1016/j.drugpo.2020.102997.

Goode E. (2008) Moral panics and disproportionality: The case of LSD use in the sixties. Deviant Behavior 29: 533-543.

Griffiths R, Richards W, McCann U, et al. (2006) Psilocybin can occasion mystical-type experiences having substantial and sustained personal meaning and spiritual significance. Psychopharmacology 187: 268-283.

Griffiths RR, Johnson MW, Richards WA, et al. (2011) Psilocybin occasioned mystical-type experiences: immediate and persisting dose-related effects. Psychopharmacology 218: 649-665.

Grof S. (1980) LSD psychotherapy, Alameda: Hunter House.

Gurholt KP and Broch TB. (2019) Outdoor life, nature experience, and sports in Norway: tensions and dilemmas in the preservation and use of urban forest. Sport in Society 22: 573-588.

Haber AF. (2009) Animism, relatedness, life: Post-Western perspectives. Cambridge Archeological Journal 19: 418-430.

Hendricks P, Thorne C, Clark C, et al. (2015) Classic psychedelic use is associated with reduced psychological distress and suicidality in the United States adult population. Journal of Psychopharmacology 29: 280-288.

Holstein J and Gubrium J. (2000) The self we live by: Narrative identity in a postmodern world. , New York: Oxford University Press. 
Hood R, Hill P and Spilka B. (2009) The psychology of religion, New York: Guilford Press. Hornby AS. (1974) Oxford Advanced Dictionary, Oxford: Oxford University Press.

Hufford D. (2005) Sleep paralysis as spiritual experience. Transcultural Psychiatry 42: 11-45.

Hysek C, Schmid Y and Simmler L. (2014) MDMA enhances emotional empathy and prosocial behavior. Social Cognitive and Affective Neuroscience 9: 1645-1652.

James W. (1994 (1902)) The varieties of religious experience, New York: Random House.

Jantzen G. (1995) Power, gender and Christian mysticism, Cambridge: Cambridge University Press.

Johnson M and Griffiths R. (2017) Potential therapeutic effects of psilocybin.

Neurotherapeutics 14: 734-740.

Johnstad P, G. (2020) A dangerous method? Psychedelic therapy at Modum Bad, Norway, 1961-76. History of Psychiatry 31: 217-226.

Johnstad P, G. (2018) Powerful substances in tiny amounts: An interview study of psychedelic microdosing. Nordic Studies on Alcohol and Drugs 35: 39-51.

Katz S. (1978) Mysticism and philosophical analysis, New York: Oxford University Press.

Kelly E and Kelly EW. (2007) Irreducible mind. Towards a psychology for the 21st century, Lanham: Rowman \& Littlefield.

Kvale S and Brinkmann S. (2009) Interviews: learning the craft of qualitative interviewing, London: Sage.

Kyzar E, Nichols DE and Gainetdinov R. (2017) Psychdelic drugs in biomedicine. Trends in Pharmacological Sciences 38: 992-1005.

Langlitz N. (2013) Neuropsychedelia, Berkeley: University of California Press.

Leary T, Metzner R and Alpert R. (1964) The psychedelic experience, London: Penguin.

Lerner M and Lyvers M. (2006) Values and beliefs of psychedelic drug users: A crosscultural study. Journal of Psychoactive Drugs 38: 143-147. 
Liechti ME. (2017) Modern clinical research on LSD. Neuropharmacology 42: 2114-2127.

Lloyd D, Lewis E and Payne J. (2012) A qualitative analysis of sensory phenomena induced by perceptual deprivation. Phenomenology and the Cognitive Sciences 11: 95-112.

Loseke DR. (2007) The study of identity as cultural, institutional, organizational, and personal narratives: Theoretical and empirical integrations. Sociological Quarterly 48: 661-688.

Lyons T and Carhart-Harris RL. (2018) Increased nature relatedness and decreased authoritarian political views after psilocybin for treatment-resistant depression. Journal of Psychopharmacology 32: 811-819.

MacLean K, Johnson M and Griffiths, R. (2011) Mystical experiences occasioned by the hallucinogen psilocybin lead to increases in the personality domain of openness. Psychopharm 25: 1453-61.

McKenna T. (1992) Food of the gods, New York: Bantam Books.

Nichols DE. (2016) Psychedelics. Pharmacological Reviews 68: 264-355.

Norwegian Institute of Public Health. (2018) Drugs in Norway, Oslo: National Institute of Public Health.

Oram, M. (2014). Efficacy and enlightenment: LSD psychotherapy and the drug amendments of 1962. Journal of the History of Medicine and Allied Science 69: 221-250.

Perrone D. (2016) Polysubstance use profiles among electronic dance music party attendees in New York City and their relation to use of new psychoactive substances. In: Brroks A (ed) Drugs, clubs and young people. London: Routledge, 40-63.

Pollan M. (2018) How to change your mind. The new science of psychedelics, London: Allen Lane.

Presser L. (2009) The narratives of offenders. Theoretical Criminology 13: 177-200. 
Riley S, Thompson J and Griffin C. (2010) Turn on, tune in, but don't drop out: The impact of neoliberalism on magic mushroom users' (in)ability to imagine collectivist social worlds. International Journal of Drug Policy 21: 445-451.

Rucker JJH, Iliff J and Nutt D. (2018) Psychiatry \& the psychedelic drugs. Past, present \& future. Neuropharmacology 142: 200-218.

Sandberg S. (2013) Are self-narratives strategic or determined, unified or fragmented? Reading Breivik's Manifesto in light of narrative criminology. Acta Sociologica 56: 69-83.

Sandberg S and Tutenges S. (2015) Meeting the djinn: Stories of drug use, bad trips, and addiction. In: Presser L and Sandberg S (eds) Narrative Criminology. New York: New York University Press.

Scultes RE and Hofmann A. (1979) Plants of the gods, New York: McGraw-Hill.

Silverman D. (2009) Doing qualitative research, London: Sage.

Skogen K, Helland H and Kaltenborn B. (2018) Concern about climate change, biodiversity loss, habitat degradation and landscape change: Embedded in different packages of environmental concern? Journal for nature conservation 44: 12-20.

Smith R. (2016) The virtues of unknowing. Journal of Philosophy of Education 50: 272-284. Stace WT. (1987 (1969)) Mysticism and philosophy, New York: Oxford University Press.

Storr W. (2019) The science of storytelling, London William Collins.

Stringer C. (2003) Human evolution: Out of Ethiopia. Nature 423: 692-695.

Tiryakian E. (1995) Collective effervescence, social change and charisme: Durkheim, Weber and 1989. Intwernational Sociology 10: 269-281.

Wasserstrom S. (1999) Religion after religion, Princeton: Princeton University Press.

Wasson RG, Hofmann A, Ruck CAP, et al. (2008) The road to Eleusis, New York: North Atlantic Books. 
Watts A. (1968) Psychedelics and Religious Experience California Law Review 56: 74-85.

Webb M, Copes H, Hendricks PS (2019) Narrative identity, rationality and microdosing classic psychedelics. International Journal of Drug Policy 70: 33-39.

Wesson DR. (2011) Psychedelic drugs, hippie counterculture, speed and phenobarbital treatment of sedative-hypnotic dependence: a journey to the Haight Ashbury in the Sixties. Journal of Psychoactive Drugs 43: 153-164.

\footnotetext{
${ }^{i}$ Let us give a brief overview of the substances described: LSD is short for Lysergic acid diethylamide, also known as acid. Psilocybin is a naturally occurring psychedelic compound, produced by more than 200 species of mushrooms. Ayahusaca is a Peruvian brew made out of psychedelic ingredients from plants of the Amazon basin, and used in spiritual ceremonies. DMT (N,N-Dimethyltryptamine) is a chemical substance occurring in many plants which has been used in many cultures for ritual purposes. 2C-B (2,5-dimethoxy-4bromophenethylamine) is a psychedelic drug of the 2C-family, first synthesized in 1974. MDMA (3,4-Methyl enedioxymethamphetamine) is commonly known as ecstasy (E), primarily used for recreational purposes. Most participants in our study did not recognize MDMA as a "real" psychedelic.
} 